\title{
Comportamientos sociales y sexuales asociados a las infecciones de transmisión sexual en jóvenes del Alto Huallaga
}

\author{
Social and sexual behaviors associated to youngsters' sexually transmitted infections \\ in Alto Huallaga, Peru
}

\author{
Walter Gómez ${ }^{1}$, Bernardo Damaso ${ }^{2}$, Carlos Cortegana ${ }^{3}$, Pedro Lahura ${ }^{4}$, Juan Motta ${ }^{5}$ \\ ${ }^{1}$ Doctorado Ciencias de la Salud, Mg. Salud y Seguridad Social, UNMSM. Hospital Tingo María, EsSalud, Huánuco, Perú. \\ 2 Médico Internista, Epidemiólogo Clínico, Hospital II Huánuco, EsSalud, Huánuco, Perú. \\ ${ }^{3}$ Biólogo. Hospital Tingo María, MINSA, Tingo María, Huánuco, Perú. \\ ${ }^{4}$ Médico Cirujano. Hospital II Huánuco, EsSalud, Huánuco, Perú. \\ ${ }^{5}$ Médico Patólogo. Hospital I Tingo María, EsSalud, Huánuco, Perú.
}

Resumen

Objetivo: Determinar la asociación de los comportamientos sociales y sexuales con las infecciones de transmisión sexual (ITS/ $\mathrm{VIH} /$ sida), en jóvenes del Alto Huallaga, 2004. Diseño: Estudio de casos y controles. Lugar: Establecimientos de salud del Alto Huallaga. Participantes: Jóvenes de 18 a 24 años. Intervenciones: Se estudió jóvenes que presentaron alguna ITS VIH/sida, durante el año 2004, realizándose análisis estadístico bivariado (prueba de chi cuadrado y prueba exacta de Fisher) y análisis multivariado (regresión logística). En el procesamiento y análisis de los datos, se utilizó el programa estadístico Stata versión 9, con apoyo del EPIDAT 3,1. Principales medidas de resultados: Edad, características familiares, vida sexual y asociación con ITS/VIH/sida. Resultados: De 1518 sujetos de investigación, 506 fueron casos con antecedente de infección de transmisión sexual y 1012 los controles, sin antecedente de ITS; $921(60,7 \%)$ fueron de sexo femenino, $29 \%$ procedían de Rupa Rupa y $64 \%$ tenía entre 21 a 24 años. Al análisis bivariado, al igual que el análisis de regresión logística, resultaron estar asociados a las variables sexo (femenino: OR 2,40 (IC 95\% 1,83 a 3,14)), edad (rango de 21 a 24 años; OR 1,38 (IC 95\% 1,08 a 1,78)), tipo de amigos (pandillaje; OR 1,19 (IC 95\% 1,05 a 1,35), tipo de relación padre - hijo (no dialogan; OR 1,28 (IC $95 \% 1,12$ a 1,46)), edad de primera relación sexual (menores de 14 años; OR 1,09 (IC 95\% 1,02 a 1,15), número de parejas sexuales (dos o más; OR 4,74 (IC 95\% 3,31 a 6,80). Conclusiones: La edad de 21 a 24 años, el grupo de pandillaje, los padres e hijos que no conversan, la primera relación sexual en menores de 14 años y el tener dos o más parejas sexuales fueron las variables asociadas a las infecciones de transmisión sexual $\mathrm{VIH} / \mathrm{sida}$.

Palabras clave: Enfermedades de transmisión sexual; adolescente; parejas sexuales: infecciones por VIH.
Abstract

Objective: To determine social and sexual behaviors associated to sexually transmitted diseases (STD/HIV/AIDS) in youngsters from Alto Huallaga, Peru, in 2004. Design: Case-control study. Setting: Alto Huallaga's health establishments. Participants: Youngsters 18 to 24 year-old. Interventions: Youngsters 18 to 24 year-old with STD/HIV/AIDS were studied. Bivariable statistics analysis consisted in chi-square test and Fisher's test. Multivariable statistic analysis used logistic regression. For data prosecution and analysis we used STATA v9 and EPIDAT 3,1 statistic software. Main outcome measures: Age, family characteristics, sexual history and their association with STD/HIV/AIDS. Results: From 1518 subjects, 506 cases had sexually transmitted infection history and 1012 controls did not have STD antecedent; 921 (60,7\%) were females, $29 \%$ came from Rupa Rupa, and $64 \%$ were 21 to 24 year-old. Both bivariate analysis and logistic regression analysis were associated to sex (female; OR 2,40 (95\% Cl 1,83-3,14)), age ( 21 to 24 years; $O R \quad 1,38(95 \% \mathrm{Cl} 1,08-1,78)$ ), types of friends (gang group; OR 1,19 (95\% Cl 1,05 - 1,35)), relationship between parents

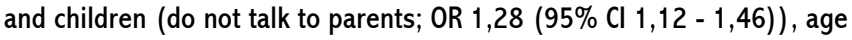
at sexual initiation (less than 14 year-old; OR $1,09(95 \% \mathrm{Cl} 1,02-1,15)$ ), number of sexual partners (two or more; OR 4,74 (95\% Cl 3,31-6,80)). Conclusions: The 21 to 24 years old group, gang group, parents and children that do not dialogue, first sexual relationship when less than 14 year-old, and having two or more sexual partners were the variables associated to sexually transmitted infections/ HIV/AIDS.

Key words: Sexually transmitted diseases; adolescent; sexual partners; HIV infections.

\section{INTRODUCCIÓN}

En el mundo se observa una tendencia ascendente en la ocurrencia de las infecciones de transmisión sexual (ITS), incluido el $\mathrm{VIH} /$ sida, a expensas de un desplazamiento de las relaciones sexuales a edades cada vez más tempranas ${ }^{(1,2)}$. Los jóvenes están creciendo en un ambiente de cambios dinámicos. Se estima que anualmente ocurren alrededor de 33 millones de casos nuevos de infección de transmisión sexual a nivel mundial; alrededor de $85 \%$ de estos casos ocurre en países en desarrollo ${ }^{(3)}$.

Las ITS han permanecido durante varias décadas dentro de las primeras 5 causas por las cuales las personas buscan servicio de salud, presentándose el mismo fenómeno en el Perú ${ }^{(4-11)}$. En los distritos de Tocache, Uchiza, Santa Lucía, Aucayacu, Rupa Rupa y Puerto Inca-Súngaro, que corresponden a la provincia de Leoncio Prado y Tocache, departamentos de Huánuco y San Martín, respectivamente, se viene dando un incremento sostenido de estas enfermedades, básicamente en la población de jóvenes (18 a 24 años), debido a la expansión de determinados comportamientos sexuales y sociales de riesgo para las ITS, que incluye al VIH/sida. Este proceso viene distorsionando el ejercicio connatural de la sexualidad humana, potenciada por los problemas estructurales de pobreza y pobreza extrema, en estos ámbitos sociogeográficos del Alto Huallaga, y reforzado por los problemas de la erradicación del narcotráfico y el facilismo del modus vivendi de los jóvenes de esta zona. Es importante resaltar que la asociación de las variables que se estudia en el presente trabajo con las ITS, ya ha sido demostrada en otros estudios ${ }^{(12)}$. El presente se refiere a un contexto sociocultural de selva alta, donde el ejercicio de la sexua- 
lidad humana responde a características particulares que inciden en el incremento de las infecciones de transmisión sexual en los jóvenes y que es necesario intervenir para modificar el comportamiento de estas enfermedades en estos escenarios. Hemos considerado para este estudio las variables edad, tipo de familia, amigos, número de parejas sexuales, relaciones sexuales, tipo de relación sexual; no consideramos los factores como drogadicción, delincuencia, comercio de drogas y trabajo sexual, debido a que, según reportes de la Dirección Regional de Salud de Huánuco (Red Leoncio Prado - Alto Huallaga), son identificados como problemas en número escaso (no determinado) en nuestra región ${ }^{(13)}$. Sin embargo, la identificación del trabajo sexual debería ser planteada en estudios futuros, al ser un problema particular del género femenino.

El objetivo de presente estudio fue determinar los comportamientos sociales y sexuales asociados a la infección por transmisión sexual (incluido el VIH/sida) en los jóvenes del Alto Huallaga, 2004. Esta investigación podría ser una contribución para intervenir el problema de las infecciones de transmisión sexual en los jóvenes del Alto Huallaga, con un alto nivel de eficacia en los escenarios socio geográficos incluidos en el estudio, a partir del conocimiento generado, así como prevenir el daño que puedan causar en los jóvenes, a través de la modificación de estos comportamientos sociales y sexuales inadecuados, mejorando por lo tanto la calidad de vida de nuestra población afectada.

\section{MÉTODOS}

Se realizó un estudio de tipo de casos y controles, con el criterio de semejanza de ecosistemas y de perfiles epidemiológicos respecto a las infecciones de transmisión sexual. Los sujetos de estudio fueron los jóvenes de 18 a 24 años que presentaron ITS (incluido VIH/sida), en el año 2004, que tuvieron diagnóstico sindrómico a nivel genital, en los establecimientos de salud a los cuales asistieron, en los distritos Tocache, Uchiza, Santa Lucía, Aucayacu, Rupa Rupa y Puerto Inca Súngaro.

El tamaño de muestra, teniendo en cuenta un nivel de confianza de $95 \%$, poder $80 \%$, relación no expuestos-expuestos de 2:1, porcentaje de exposición de casos de $61 \%$ (445 casos) y porcentaje de exposición controles 53\% (935 controles). El total de la muestra fue calculada en 1380 jóvenes, considerándose el $10 \%$ adicional por existir la probabilidad de no hallar algunos casos y controles en el proceso de la recolección de datos. A una muestra probabilística de estos jóvenes, se aplicó una encuesta estructurada, previo consentimiento informado. Se definió operacionalmente la variable ITS como el diagnostico sindrómico a nivel de genitales, expresado por el participante del estudio, que requirió tratamiento en los establecimientos de salud. La variable tipo de familia (¿Cómo considera a su familia?) fue categorizada en 'unida', 'desintegrada', 'conflictiva' y 'cada uno vive su vida' (fue recategorizada con 'unida' y 'no unida'). La variable tipo de amigos (¿Cómo clasificarías a tus amigos?) fue categorizada en 'deportistas', 'participa en grupos artísticos o culturales', 'participa en agrupaciones religiosas', 'no trabaja/no estudia' o 'pandillaje' (se recategorizó en 'pandillaje y no trabaja' y 'participa en agrupaciones'). La variable número de parejas sexuales (¿Cuántas parejas sexuales tienes actualmente?) fue categorizada en 'uno', 'mas de uno' y 'ninguno' (se recategorizó en 'dos o más parejas' y 'una o ninguna pareja'). La variable relaciones sexuales cuando no se cuenta con método anticonceptivo (si estás con tu pareja y vas a tener relaciones sexuales, pero no cuentas con ningún método de protección, ¿qué haces?) fue categorizada en 'tenemos relaciones sexuales' y 'no tenemos relaciones sexuales'. La variable tipo de relación sexual (¿Qué tipos de relaciones sexuales practicas ahora?) fue categorizada en 'heterosexual', 'homosexual' y 'otros' (se recategorizó en 'heterosexual' y 'homosexual y otros').

Para el análisis estadístico, se realizó un análisis univariado y bivariado, utilizando la prueba chi cuadrado y la prueba exacta de Fisher, para variables cualitativas. Se realizó el análisis de regresión logística para la infección de transmisión sexual, considerándose un $p<0,05$ como significativo. En el procesamiento y análisis de los datos, se utilizó el programa estadístico Stata versión 9, con apoyo del EPIDAT 3,1 y Microsoft Excel 2003.

\section{RESULTADOS}

Un total de 1518 jóvenes ingresaron al estudio, 915 de establecimientos del Ministerio de Salud y 603 de establecimientos de EsSalud; 921 jóvenes (60,7\%) fueron de sexo femenino, siendo el rango de edad más frecuente de 21 a 24 años (966 jóvenes, $63,6 \%$ ) y el mayor número captado correspondió al distrito de Rupa Rupa (Tingo Maria, con 29,3\%). Del total de la población, $506(33,3 \%)$ tuvieron antecedente de infección de transmisión sexual.

En la Tabla 1 se muestra el análisis inferencial bivariado de las características demográficas y el antecedente de ITS.

En la Tabla 2, se presenta la distribución de las variables sociales con el antecedente de infección de transmisión sexual. Se ha considerado las variables tipo de familia, tipo de amigos y tipo de relación entre padres e hijos.

Tabla 1. Características demográficas en jóvenes con antecedente de infección de transmisión sexual (ITS) en zonas del Alto Huallaga, 2004. ( $n=1518)$

\begin{tabular}{|c|c|c|c|c|c|c|}
\hline \multirow{3}{*}{ Características demográficas } & \multicolumn{4}{|c|}{ Antecedente de ITS } & \multirow{3}{*}{$p$} & \multirow{3}{*}{ OR (IC 95\%) } \\
\hline & \multicolumn{2}{|c|}{$\mathrm{Si}$} & \multicolumn{2}{|c|}{ No } & & \\
\hline & $\mathrm{n}$ & $\%$ & $\mathrm{n}$ & $\%$ & & \\
\hline \multicolumn{7}{|l|}{ Sexo } \\
\hline Masculino & 126 & 21,1 & 471 & 78,9 & 0,000 & $0,38(0,29 ; 0,48)$ \\
\hline Femenino & 380 & 41,3 & 541 & 58,7 & & \\
\hline \multicolumn{7}{|l|}{ Edad } \\
\hline 18 a 20 años & 160 & 29,0 & 392 & 71,0 & 0,006 & $0,73(0,57 ; 0,92)$ \\
\hline 21 a 24 años & 346 & 35,8 & 620 & 64,2 & & \\
\hline
\end{tabular}

Tabla 2. Características sociales en jóvenes con antecedente de infección de transmisión sexual (ITS) en zonas del Alto Huallaga, 2004. ( $n=1518)$

\begin{tabular}{|c|c|c|c|c|c|c|}
\hline \multirow{3}{*}{ Características demográficas } & \multicolumn{4}{|c|}{ Antecedente de ITS } & \multirow{3}{*}{$p$} & \multirow{3}{*}{ OR (IC 95\%) } \\
\hline & \multicolumn{2}{|c|}{$\mathrm{Si}$} & \multicolumn{2}{|c|}{ No } & & \\
\hline & $\mathrm{n}$ & $\%$ & $\mathrm{n}$ & $\%$ & & \\
\hline \multicolumn{7}{|l|}{ Tipo de familia } \\
\hline No unida & 240 & 38,0 & 391 & 62,0 & \multirow[t]{2}{*}{0,001} & \multirow[t]{2}{*}{$1,43(1,14 ; 1,78)$} \\
\hline Unida & 266 & 30,0 & 621 & 70,0 & & \\
\hline \multicolumn{7}{|l|}{ Tipo de amigos } \\
\hline Pandillaje, no trabaja & 50 & 69,4 & 22 & 30,6 & \multirow[t]{2}{*}{0,000} & \multirow[t]{2}{*}{$4,93(2,88 ; 8,65)$} \\
\hline Agrupación social & 456 & 31,5 & 990 & 68,5 & & \\
\hline \multicolumn{7}{|l|}{ Tipo de relación entre padres e hijos } \\
\hline No converso, agresivo & 194 & 38,8 & 306 & 61,2 & \multirow[t]{2}{*}{0,001} & \multirow[t]{2}{*}{$1,43(1,13 ; 1,80)$} \\
\hline Converso con ellos & 312 & 30,6 & 706 & 69,4 & & \\
\hline
\end{tabular}


En la Tabla 3, se presenta la distribución de las variables sexuales con el antecedente de infección de transmisión sexual. Se ha considerado las variables edad de la primera relación sexual, número de parejas sexuales, tipo de la relación sexual a la actualidad y la realización del coito, si se cuenta con un método anticonceptivo.

En la Tabla 4 se presenta la significancia estadística bivariada asociada al antecedente de infección de transmisión sexual, encontrándose como importantes al sexo, edad, tipo de familia, tipo de amigos, tipo de relación entre padres e hijos, edad de la primera relación sexual, número de parejas sexuales y la realización del coito, si se
Tabla 3. Características sexuales en jóvenes con antecedente de infección de transmisión sexual (ITS) en zonas del Alto Huallaga, 2004. ( $n=1518)$

\begin{tabular}{|c|c|c|c|c|c|c|}
\hline \multirow{3}{*}{ Características demográficas } & \multicolumn{4}{|c|}{ Antecedente de ITS } & \multirow{3}{*}{$p$} & \multirow{3}{*}{ OR (IC 95\%) } \\
\hline & \multicolumn{2}{|c|}{$\mathrm{Si}$} & \multicolumn{2}{|c|}{ No } & & \\
\hline & $\mathrm{n}$ & $\%$ & $\mathrm{n}$ & $\%$ & & \\
\hline \multicolumn{7}{|l|}{ Edad de la primera relación sexual } \\
\hline 13 o menos edad & 42 & 18,7 & 183 & 81,3 & 0,000 & $1,41(0,28 ; 0,58)$ \\
\hline 14 o mas edad & 506 & 37,9 & 829 & 62,1 & & \\
\hline \multicolumn{7}{|l|}{ Número de parejas sexuales } \\
\hline Dos o mas & 108 & 63,2 & 63 & 36,8 & 0,000 & $4,08(2,89 ; 5,79)$ \\
\hline Uno o ninguno & 398 & 29,5 & 949 & 70,5 & & \\
\hline \multicolumn{7}{|c|}{ Tipo de relación sexual a la actualidad } \\
\hline Homosexual y otros & 18 & 32,1 & 38 & 67,9 & 0,847 & $0,94(0,50 ; 1,71)$ \\
\hline Heterosexual & 488 & 33,4 & 974 & 66,6 & & \\
\hline \multicolumn{7}{|l|}{ RS cuando no se cuenta con MAC } \\
\hline Si realiza RS & 234 & 46,2 & 272 & 53,8 & 0,000 & $1,48(1,18 ; 1,85)$ \\
\hline No realiza RS & 371 & 36,7 & 641 & 63,3 & & \\
\hline
\end{tabular}

RS: relación sexual

MAC: métodos anticonceptivos
Tabla 4. Análisis bivariado de características demográficas, sociales y sexuales con antecedente de infección de transmisión sexual en zonas del Alto Huallaga, 2004. $(n=1518)$

\begin{tabular}{lrrr}
\hline \multicolumn{1}{c}{ Variables } & Chi2 & $p$ & OR (IC 95\%) \\
\hline Sexo & 66,21 & 0,000 & $0,38(0,29 ; 0,48)$ \\
Edad & 7,38 & 0,006 & $0,73(0,57 ; 0,92)$ \\
Tipo de familia & 10,74 & 0,001 & $1,43(1,14 ; 1,78)$ \\
Tipo de amigos & 44,35 & 0,000 & $4,93(2,88 ; 8,65)$ \\
Tipo de relación entre padres e hijos & 10,03 & 0,001 & $1,43(1,31 ; 1,80)$ \\
Edad de la primera relación sexual & 25,57 & 0,000 & $0,41(0,28 ; 0,58)$ \\
& & & \\
Número de parejas sexuales & 77,14 & 0,000 & $4,08(2,89 ; 5,79)$ \\
RS cuando no se cuenta con MAC & 12,93 & 0,000 & $1,48(1,18 ; 1,85)$ \\
Tipo de RS a la actualidad & 0,04 & 0,847 & $0,94(0,50 ; 1,71)$ \\
\hline
\end{tabular}

RS: relación sexual

MAC: métodos anticonceptivos el tipo de familia (no unida, OR 1,32; IC 95\% 1,05 a 1,65), el número de parejas sexuales (dos o más parejas sexuales, OR 4,74; IC 95\% 3,31 a 6,80$)$; la variable tipo de amigos (pandillaje y no trabajar) es una variable de interacción negativa para el tipo de familia, que en el análisis bivariado resulta de significancia estadística.

cuenta con un método anticonceptivo. El tipo de la relación sexual practicado a la actualidad no hallo diferencia estadísticamente significativa.

En el análisis de regresión logística, se encontró asociación estadísticamente significativa entre el antecedente de infección de transmisión sexual con la edad, tipo de familia y el número de parejas sexuales.

El análisis multivariado (Tabla 5) muestra las variables con asociación estadística significativa con el antecedente de infección de transmisión sexual: la edad (entre 18 y 20 años, OR 1,32; IC95\% 0,51 a 0,81),

Tabla 5. Análisis multivariado en los jóvenes con antecedente de infección de transmisión sexual en zonas del Alto Huallaga, 2004. ( $n=1518)$

\begin{tabular}{lrccr}
\multicolumn{1}{l}{ Modelo } & \multicolumn{1}{c}{ Z } & & & \\
\hline \multicolumn{1}{c}{ Variables } & $-3,61$ & 0,000 & 0,65 & $(0,51 ; 0,81)$ \\
\hline Edad & 2,46 & 0,014 & 1,32 & $(1,05 ; 1,65)$ \\
Tipo de familia & 8,28 & 0,000 & 4,19 & $(2,98 ; 5,87)$ \\
Número de parejas sexuales & & & & \\
Tipo de amigos* & & & & \\
\hline
\end{tabular}

* variable de interacción negativa con la variable tipo de familia

Validando el modelo:

número de observaciones

número de grupos

Hosmer-Lemeshow chi2(6)

Prob $>$ chi2
Se presenta un modelo predictivo:

ETS $=-0,83-0,43($ edad $)+0,28($ tipo de familia) + 1,43 (número parejas sexuales)

\section{DISCUSIÓN}

Las infecciones de transmisión sexual constituyen un importante problema de salud pública en nuestro país y a nivel mundial ${ }^{(8)}$. Un elevado número de personas adquiere una de ellas o sufre una complicación de una ITS no tratada oportuna y adecuadamente, o se infectan por el virus de la inmunodeficiencia humana (VIH) ${ }^{(8)}$, causante del síndrome de inmunodeficiencia adquirida (sida), como consecuencia de la presencia de una ITS ${ }^{(6,7)}$.

Las complicaciones de las ITS ocasionan una enorme carga de morbilidad y mortalidad, especialmente en adultos en edad reproductiva, pero afectando también a los recién nacidos. Entre las complicaciones más importantes tenemos: enfermedad pélvica inflamatoria, embarazo ectópico, abortos, infertilidad, sífilis congénita, parto prematuro, restricción del crecimiento fetal intrauterino, entre otros ${ }^{(6-8)}$. Además, la importancia de las ITS se ha incrementado con el reconocimiento de su rol facilitador en la transmisión del VIH, incremento en la probabilidad de transmitir o de adquirir el VIH cuando existe una ITS en la persona infectada o en la que se expone, respectivamente ${ }^{(6-8)}$.

La tendencia al incremento de casos de las infecciones de transmisión sexual, incluido el VIH/sida en el mundo, es sostenida a expensas de un desplazamiento de las relaciones sexuales a edades cada vez más tempranas, como se informa en un estudio peruano ${ }^{(14,15)}$. Los jóvenes están creciendo en un ambiente de cambios dinámicos. Se estima que anualmente ocurren alrededor de 33 millones de casos nuevos de infección de transmisión sexual, a nivel mundial; alrededor de $85 \%$ de estos casos ocurre en países en desarrollo ${ }^{(3)}$. Las ITS han permanecido durante varias décadas dentro de las primeras 5 causas por las cuales las personas buscan servicio de salud, presentándose el mismo fenómeno en el Perú ${ }^{(4-11)}$. En los distritos de Tocache, Uchiza, Santa Lucía, Aucayacu, Rupa Rupa y Puerto Inca Sungaro, que corresponden a la provincia de Leoncio Prado y Tocache, departamentos de Huánuco y San Martín, se viene dando un incremento sostenido de estas enfermedades, básicamente en la población de jóvenes (18 a 24 años), debido a la expansión de determinados comportamientos sociales y sexuales de riesgo para las ITS. La prevalencia de algunas infecciones de transmisión sexual en mujeres adolescentes es alta. En un estudio realizado por el Programa de Control de En- 
fermedades de Transmisión Sexual y sida del Ministerio de Salud del Perú, en el año 2000, se encontró que, en mujeres menores de 20 años, la prevalencia de infección cervical por Chlamydia trachomatis en el Hospital Dos de Mayo fue $25,7 \%$, mientras que en las mujeres mayores de 20 o más años de edad fue $9,1 \%$ (14). Existen condiciones fisiológicas para que las infecciones de transmisión sexual se produzcan con mayor frecuencia ${ }^{(16)}$. A nivel latinoamericano, un estudio chileno determina la prevalencia de ITS por Chlamydia trachomatis, no encontrándose ningún caso de Neisseria gonorrhoeae ${ }^{(17)}$.

Las mujeres adolescentes constituyen el 22,8\% de la población en el Perú y están en riesgo de adquirir infecciones de transmisión sexual y el VIH, debido a su desventaja de género ${ }^{(2,8,18,19)}$, la precocidad sexual cada vez mayor ${ }^{(4,17,20)}$, su tendencia a exponerse a riesgos, el bajo nivel socioeconómico, la falta de conocimientos sobre sexualidad, el escaso uso de condón ${ }^{(21,22)}$ y el desconocimiento de la actividad sexual de su pareja ${ }^{(19,23)}$ o la negación de padecer una infección de transmisión sexual ${ }^{(2)}$. Esta asociación inicialmente demostrada en el análisis bivariado no se mantuvo en el análisis multivariado.

Con respecto a la asociación entre la variable presencia de infección de transmisión sexual y variable edad, se encuentra relación significativa en el análisis multivariado con el rango de 21 a 24 años de edad. Este resultado es coincidente con comunicaciones de diferentes estudios realizados, donde las mujeres están en mayor riesgo de presentar una ITS $(2,8,14,17,19,23,24)$. En otras realidades, los cambios de conducta sexual se inician desde los 15 años de edad ${ }^{(1,8)}$.

La asociación entre la variable presencia de infección de transmisión sexual y variable edad de la primera relación sexual, se encuentra relación significativa solo en el análisis bivariado y no en el multivariado. Este resultado es observado en los reportes africanos ${ }^{(24)}$. Un aspecto resaltante de este grupo etáreo es su baja consideración a presentar una infección de transmisión sexual, por lo que no existe un cambio en los factores de riesgo para ITS ${ }^{(17,23)}$.

La asociación entre la variable presencia de infección de transmisión sexual y variable sexo es significativa en el análisis bivariado, no persistiendo esta asociación en el análisis multivariado. Este resultado es descrito en comunicaciones americanas, que establece al empleo del sexo como mercancía para obtener drogas o dinero, aumentando la incidencia de casos con
VIH e infecciones de transmisión sexual, predominando esta actitud en el sexo femenino ${ }^{(8,19,25,26)}$. Así mismo, el empleo de los métodos anticonceptivos, como el condón, depende de las características personales del individuo ${ }^{(8,19,27)}$.

La asociación entre la variable presencia de infección de transmisión sexual y variable tipo de familia, se encuentra relación significativa en el análisis multivariado. Al realizar el análisis estratificado, se encuentra que el subgrupo 'familia no unida' se relaciona con la infección de transmisión sexual. Nuestro hallazgo es coincidente con estudios que demuestran que la violencia familiar condicionante de estrés psicológico, traumatismos ginecológicos, promueven las infecciones de transmisión sexual ${ }^{(28)}$. El estudio colombiano de Mosquera y col demuestra que la relación con los padres influye en los conocimientos, actitudes y prácticas del empleo de métodos anticonceptivos relacionados directamente con las ITS ${ }^{(29)}$. Nuestros resultados revelan el papel importante de la familia en el comportamiento sexual de los adolescentes y resaltan la diferencia con otros estudios anteriores, donde se subestima los factores contextuales del hogar. La erosión de la familia tradicional, en la sociedad occidental actual, ha contribuido a crear condiciones que favorecen las ITS y mayor fecundidad en la adolescencia ${ }^{(30)}$.

Respecto a la asociación entre la variable presencia de infección de transmisión sexual y variable tipo de amigos, se encuentra relación significativa en el análisis multivariado, pero como variable de interacción o modificadora de efecto de la variable tipo de familia. Al realizar el análisis estratificado, se encuentra que los subgrupos pandillaje y no trabajar se relacionan con la infección de transmisión sexual. Este resultado es coincidente con el hallazgo americano, que evalúa a la población joven ${ }^{(25,26)}$.

La asociación entre la variable presencia de infección de transmisión sexual y variable tipo de relación entre padres e hijos, es significativa en el análisis bivariado, no persistiendo en el análisis multivariado. Al realizar el análisis estratificado, se encuentra que los subgrupos que no conversan con los padres se asocian a la infección de transmisión sexual. Este resultado es coincidente con otras comunicaciones, donde la inadecuada relación familiar, así como la violencia familiar, condicionan las infecciones de transmisión sexual, además de estrés psicológico y traumatismos físicos ginecológicos $^{(28)}$. Mosquera y col encontraron que la relación con los padres influye en los conocimientos, actitudes y prácticas del empleo de métodos anticonceptivos ${ }^{(29)}$.

Respecto a la asociación entre la variable presencia de infección de transmisión sexual y variable número de parejas sexuales, se encuentra relación significativa tanto en el análisis bivariado como en el mutivariado. Al realizar el análisis estratificado, se encuentra que los subgrupos con dos o más parejas sexuales se asocian a la infección de transmisión sexual. Nuestros resultados coinciden con varios estudios $(12,14,22,28,31)$. En otras realidades, los cambios de conductas sexuales se inician desde los 15 años de edad, así como los factores de riesgo promotores de infección de transmisión sexual ${ }^{(1)}$.

Por otro lado, al asociar la variable presencia de infección de transmisión sexual y variable relación sexual al no contar con un método anticonceptivo, se encuentra relación significativa con la decisión del coito en el análisis bivariado; sin embargo, en el multivariado se pierde esta relación. Este resultado es con un estudio realizado en adolescentes mujeres de Lima, donde concluyen que el preservativo dificultaría que el acto sexual se realice ${ }^{(12)}$. Esto evidenciaría que la mujer 'no se dejó llevar por el deseo', sino que racionalmente aceptó tener relaciones sin el método anticonceptivo. El empleo de los métodos anticonceptivos, como el condón, depende de las características personales del individuo ${ }^{(27)}$, objetivo de intervención en atención primaria de salud $^{(31,32)}$.

En conclusión del presente trabajo, los comportamientos sociales y sexuales asociados a la infección por transmisión sexual en los jóvenes del Alto Huallaga fueron la edad (rango de 21 a 34 años), tipo de familia (no unida) y el número de parejas sexuales (dos o más parejas).

\section{REFERENCIAS BIBLIOGRÁFICAS}

1. Edgardh K. Adolescent sexual health in Sweden. Sexually Transmitted Infections. 2002;78:352-6.

2. Velarde-Jurado E, Estrada-Reyes E, ErañaGuerra L, Raya-Rivera A, Velázquez-Armenta Y, Nava-Ocampo A. Sexually transmitted infections associated with vulvovaginal symptoms in adolescents denying sexual activity. Salud Pública de México. 2003;45 Sup 5:s641-6.

3. Sales JM, Milhausen RR, DiClemente RJ. A decade in review: building on the experiences of past adolescent STI/HIV interventions to optimize future prevention efforts. Sexually Transmitted Infectious. 2006;82:431-6. 
4. Chirinos J, Brindis C, Salazar VC, Bardales 0, Reátegui LR. Perfil de las estudiantes adolescentes sexualmente activas en colegios secundarios en Lima Perú. Rev Med Hered. 1999;10(2):49-61.

5. Medina R, Rechkemmer A, Garcia-Hjarles M. Prevalencia de vaginitis y vaginosis bacteriana en pacientes con flujo vaginal anormal en el Hospital Nacional Arzobispo Loayza. Rev Med Hered. 1999;10(4):144-50.

6. Ministerio de Salud del Perú. Plan de la Estrategia Sanitaria Nacional de Prevención y Control de las ITS-VIH/SIDA 2005-2009. Lima: MINSA; 2004: p. 16.

7. Ministerio de Salud. Evaluación del Plan Estratégico del MINSA, 2001-2004 para la Prevención y Control del VIH / SIDA en el Perú. Lima: MINSA; 2005: p. 78.

8. Obunge OK, Brabin L, Dollimore N, Kemp J, IkokwuWonodi C, Babatunde S, et al. A flowchart for managing sexually transmitted infections among Nigerian adolescent females. Bull World Health Organization. 2001;79:301-5.

9. Ministerio de Salud. Doctrinas, Normas y Procedimientos para el control de las ETS y el SIDA en el Perú. Lima: MINSA; 1996: p. 4.

10. OMS. Estimaciones con ETS en el Mundo. Periodo 1983 - 2001. Geneva: OMS; 2000: p 2.

11. OPS. Estimaciones de Adultos y Niños que viven con VIH/SIDA en el mundo. Washington, D.C.: OPS; 1999: p. 32.

12. Cabada M, Montoya M, Echevarría J, Samalvides $F$, Gotuzzo E. Conductas de riesgo en jóvenes que tienen contacto sexual con viajeros ("bricheros") en la ciudad del Cuzco - Perú. Rev Peru Med Exp Salud Pública. 2002;19 (2):83-6.

13. Dirección Regional de Salud, Huánuco. Análisis de la Situación de Salud de la Red Salud Leoncio Prado 2005. Tingo María: MINSA, Oficina de Epidemiología; 2005: p. 1-60.

14. Sánchez S, Atencio G, Duy N, Grande M, Flores M, Chiappe M, et al. Comparación de la educación por pares y por profesionales de la salud para mejorar el conocimiento, percepción y la conducta sexual de riesgo en adolescentes. Rev Peru Med Exp Salud Publica. 2003;20(4):206-10.

15. Portilla J, Valverde A, Romero S, Suárez M, Aliaga R, Alfaro P, et al. Prevalencia de Neisseria gonorrhoeae y Chlamydia trachomatis en gestantes atendidas en el Instituto Materno Perinatal de Lima - Perú, 1997-1998. Rev Med Exp. 1999;XV(12):25-7.
16. Brabin L, Roberts SA, Fairbrother E, Mandal D, Higgins SP, Chandiok S, Word P, Barnard G, Kitchener HC. Factors affecting vaginal pH levels among female adolescents attending genitourinary medicine clinics. Sexually Transmitted Infections. 2005;81:483-7.

17. Gaete MV, Prado VE, Altamirano PD, Martinez JB, Urrejola P, Pinto JM. Prevalence of Chlamydia trachomatis and Neisseria gonorrhoeae in Chilean asymptomatic adolescent males determined by urine simple. Sexually Transmitted Infections. 1999;75;67-8

18. Lundgren R. Protocolos de investigación para el estudio de la salud sexual y reproductiva de los adolescentes y jóvenes varones en América Latina. Washington, DC: Organización Panamericana de la Salud; 2000.

19. Nantua M, Madalena M, De Lannoy L, Ferreira A, Valeriano N. Percepción del riesgo de la infección $\mathrm{VIH}$ entre hombres y mujeres con infecciones de transmisión sexual (ITS). Ciencia y Enfermeria. 2007;XIII(1):69-80.

20. La Rosa LC. Adolescencia e iniciación sexual. Lima: Universidad Peruana Cayetano Heredia; 1997.

21. Pisan E, Cuchí P, Zacarías F, Schwartlander B, Stanecki K, Castilho E, et al. VIH y SIDA en las Américas: una epidemia multifacética. Ginebra: Organización Panamericana de la Salud. Organización Mundial de la Salud y Programa de las Naciones Unidas sobre HIV/SIDA; 2000.

22. Arnao J, Barco C, Carpio L, Castro R, Chávez V, Tapia $L$ y col. Relación de las drogas con las actitudes sexuales y vínculos de pareja. Centro de Información y Educación para la Prevención del Abuso de Drogas. Lima: CEDRO; 2002.

23. Ethier KA, Kershaw T, Niccolai L, Lewis JB, Ickovics JR. Adolescent women underestimate their susceptibility to sexually transmitted infections. Sexually Transmitted Infectious. 2003;79:408-11.

24. Ellen JM, Lane MA, McCright J. Are adolescents being screened for sexually transmitted diseases? A study of low income African American adolescents in San Francisco. Sexually Transmitted Infectious. 2000;76;94-7.

25. Edwards JM, Iritani BJ, Hallfors DD. Prevalence and correlates of exchanging sex for drugs or money among adolescents in the United Status. Sexually Transmitted Infectious. 2006;82;354-8.

26. Salazar LF, DiClemente RJ, Wingood GM, Crosby RA, Lang DL, Harrington K. Biologically confirmed sexually transmitted infection and depressive symptomatology among African-American female adolescents. Sexually Transmitted Infectious. 2006;82:55-60.

27. Crosby R, Salazar LF, DiClemente RJ. Lack of recent condom use among detained adolescent males: a multilevel investigation. Sexually Transmitted Infectious. 2004;80:425-9.

28. Stephenson R, Koenig MA, Ahmed S. Domestic violence and symptoms of gynecologic morbidity among women in North India. International Family Planning Perspectives. 2006;32(4):201.

29. Mosquera J, Mateus J. Conocimientos, actitudes y prácticas sobre métodos de planificación familiar, VIH-SIDA y el uso de los medios de comunicación en jóvenes. Colombia Médica. 2003;34(4):20612.

30. Flórez C. Factores socioeconómicos y contextuales que determinan la actividad reproductiva de las adolescentes en Colombia. Rev Panam Salud Pública/Pan Am J Public Health. 2005;18(6):388402.

31. Barros T, Barreto D, Pérez F, Santander R, Yépez E, Abad-Franch F, Aguilar M. Un modelo de prevención primaria de las enfermedades de transmisión sexual y del VIH/sida en adolescentes. Rev Panam Salud Publica/Pan Am J Public Health. 2001;10(2):86-94.

32. Gotuzzo E, Peinado J, Tijero M, Mayorca C, Badajos A. Evaluación de un programa de capacitación en educación sexual y SIDA a docentes del cono norte de Lima- Perú. 1996- 1997. Rev Med Hered. 2001;12(2):45-51.

Este estudio contó con el apoyo técnico y financiero del Proyecto Vigía "Enfrentando las Amenazas de las Enfermedades Infecciosas Emergentes y Reemergentes" (MINSA/USAID), a través del V Concurso Nacional para el Desarrollo de Estudios de Investigación en Enfermedades Infecciosas Emergentes y Reemergentes - Año 2004.

Manuscrito recibido el 6 de enero de 2008 y aceptado para publicación el 27 de febrero de 2008.

Correspondencia:

Walter Gómez Gonzáles

Hospital Tingo María, EsSalud. Jr. Bolognesi № 120, Rupa Rupa, Huánuco.

Correo-e: waltergomez29@yahoo.com

epidemio.tmaria@essalud.gob.pe 